\title{
Experimental determination of local convection heat transfer coefficient field using two-dimensional and dynamic infrared thermography (2DD-IRT) method
}

\author{
J.A. Patorski $*^{\mathrm{a}}$, F. Groeschel ${ }^{\mathrm{a}}$ \\ ${ }^{a}$ Spallation Neutron Source Div., Paul Scherrer Institute, CH-5232 Villigen-PSI, Switzerland
}

\begin{abstract}
In the scope of the Megawatt Pilot Experiment MEGAPIE, i.e. the development of a liquid metal target for a spallation neutron source, an experimental thermo-hydraulics investigation of the target proton beam entry window cooling has been performed. Goal of this investigation concerned the measurement of the local convection heat transfer coefficient (HTC) inside of the proton beam entry window area of the MEGAPIE target, in particular:

- determination of HTC absolute values,

- distribution/visualization of HTC field shape and

- dynamic behavior of HTC field i.e. visualization of HTC field fluctuations.

Within KILOPIE's experimental set-up the following conditions of MEGAPIE target have been fulfilled:

- Using of liquid metal (LM) lead-bismuth eutectic (LBE); this simultaneously serves as target material and coolant.

- Using of T91-steel; for the shell-dish of hemispherical mock-up of the proton beam entry window.

- Using of original geometry of piping insertions for the simulation of internal LBE coolant flow geometry.

In KILOPIE the improved Two-Dimensional and Dynamic of Infrared Thermography (2DD-IRT) Method, presented on Thermosense XXII in year 2000 [1,2], has been used. In this paper the improvements of 2DD-IRT method and some result of KILOPIE experimental investigations performed at PSI in Switzerland will be presented. A specially tailored Aluchrom-steel shell is used, which allows applying a uniform and known constant heat flux deposition on the outer surface of the T91-steel hemispherical mock-up of the target window. The optical non-contact IRT equipment measures the outer surface temperature of the Aluchrom-steel heater glued to the T91-steel mock-up dish. The determination of the local convection HTC is a result of ratio of the known local heat flux from the Aluchrom-steel heater to the difference between the local inner surface temperature of the T91-steel mock-up dish and the bulk temperature of the LBE coolant.
\end{abstract}

Keywords: quantitative IR thermography, MEGAPIE thermo-hydraulics, local convection heat transfer coefficient, liquid metal target, spallation neutron source

\section{INTRODUCTION}

In the scope of the long term R\&D challenge to build an Accelerator Driven System, the creation of new concepts for neutron spallation sources with high neutron flux are the subjects of world wide scientific investigations. A new concept of high power neutron spallation source with liquid metal (LM) target is developed within the international MEGAPIE Project [3]. The ultimate experimental part of the MEGAPIE project, i.e. a six months long irradiation period with an accelerated proton beam, will start mid 2006 in the spallation neutron source facility SINQ at Paul Scherrer Institute (PSI) in Switzerland. In the MEGAPIE target, the LM, in this case lead-bismuth eutectic (LBE) is simultaneously used as target material and coolant.

The general concept of the MEGAPIE target is shown in Fig. 1a. Simplifying, a system of approx. $4 \mathrm{~m}$ long concentric cylindrical pipes and vessels, is fulfilling a LBE guiding and safety functions. The LBE flow-guiding part of the target consists of two steel pipes placed in the middle of the cross section of the target. The lower part of outer pipe is closed with a hemispherical shell, forming a vertical, cylindrical liquid metal container (LMC) and causing a $180^{\circ}$-change of a downward LBE main flow $Q$ into an upward flow in the inner riser pipe (RP). The $600 \mathrm{MeV}$ proton beam comes through a lower enclosure of this system, the entry window. This window will be strongly heated; the heat deposition in the steel wall will produce a heat flux $q^{*}$ up to $140 \mathrm{~W} / \mathrm{cm}^{2}$ [4].

*jacek.patorski@psi.ch; phone +415631020 60; fax +41563103131; http://asq.web.psi.ch/ASQ/projects/liquid/liquid.html 

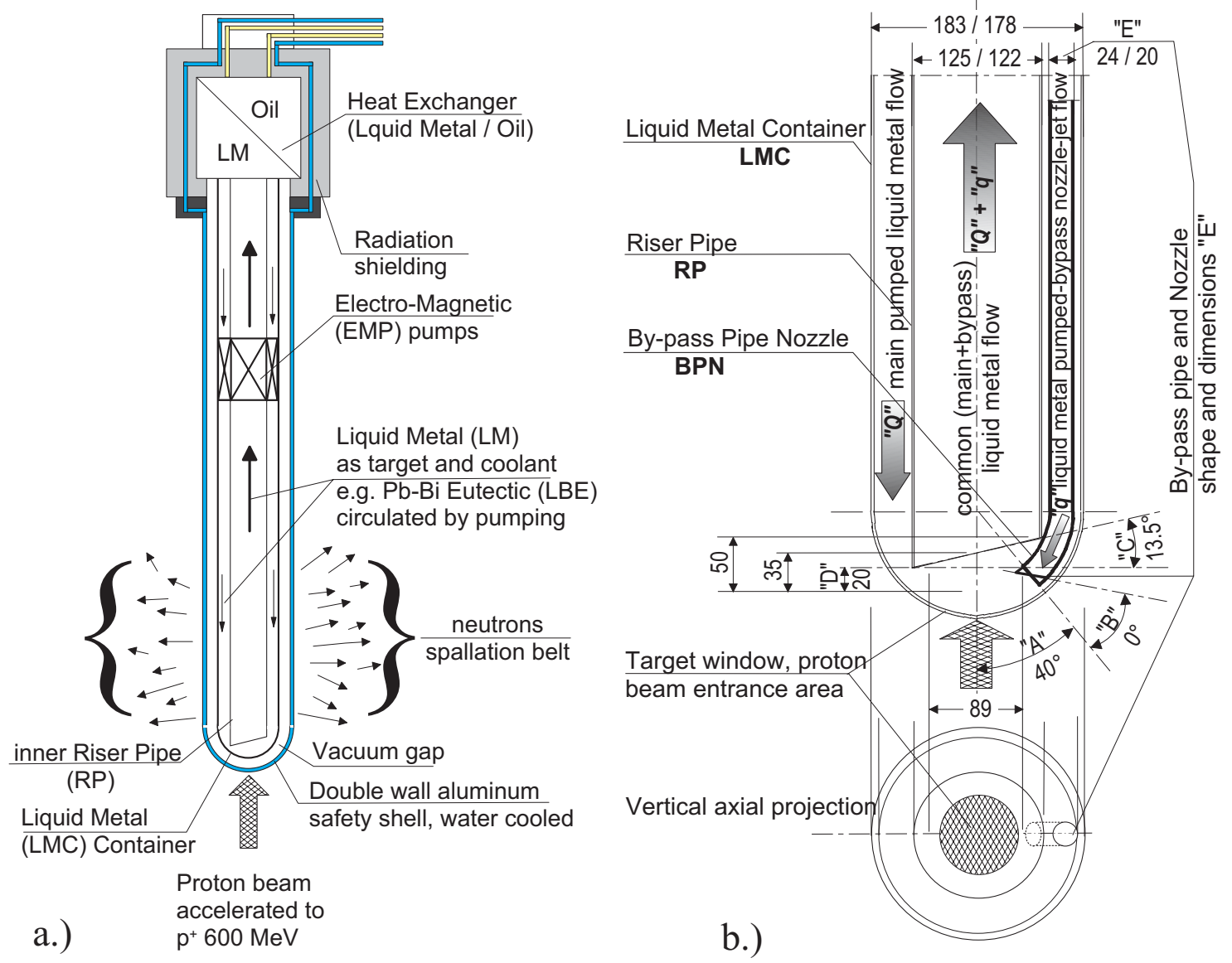

Figure 1:Schematic of MEGAPIE liquid metal target for a spallation neutron source

a.) General concept of the target in vertical configuration in SINQ facility at PSI

b.) Geometry of the lower part of the target. Principal idea of the LBE-flow configuration.

This target window is cooled by an additional bypass pipe circuit so called the by-pass pipe nozzle flow jet (BPN). The open nozzle-end of the bypass tube forms an LBE jet flow $q$ directly streaming onto the inner surface of the window.

Basic geometrical parameters of the assembled lower part of the target are displayed in Fig.1b and represent the principal idea of pumped flow configuration: main flow $Q$ and bypass jet flow $q$ providing a transporting LBE to the spallation zone and a cooling for the hemispherical proton beam entrance window. The steel wall of the window will be cooled mostly by a LBE bypass nozzle jet flow "q" across the window area and additionally by main LBE flow $Q$. The main flow $Q$ is responsible for recirculation and transporting the strongly heated LBE after spallation to the heat exchanger of the target, which is placed on the top of the whole target system.

\section{GOALS OF THE EXPERIMENTAL INVESTIGATIONS}

For a proper design and operation of the MEGAPIE target, the knowledge of the cooling characteristic of LBE flow for the specific geometrical flow configuration and for the real target window material, $\mathrm{T} 91(9 \mathrm{Cr}-1 \mathrm{MoVNb})$ martensitic steel, is necessary. Therefore we have decided to perform a series of proper thermal hydraulic experiments.

The main goal of the experiments was:

- The determination of a local convection heat transfer coefficient (HTC) over the area of the proton beam entry window for different ratios of characteristic LBE by-pass jet flow $q$ and main flow $Q$, and geometrical flow configurations.

Other objectives were:

- Visualization of the LBE jet flow cooling field for different $Q / q$ cases.

- Visualization of dynamic behavior (fluctuations) of LBE jet flow cooling field within different $Q / q$ cases. 
- Determination of optimal operating range for $q$ and $Q$, similar to the work reported in [5].

- Validation of CFD calculations.

With respect to the goals for experiments, we have simulated most conditions of MEGAPIE target: Materials (LBE and steel T91), the geometrical configuration (also with small variations labeled in Fig.1b as "A, B, C, D, E"), the bulk temperature of the LBE, local heat fluxes $q^{*}$ through the window, the main flow rate $Q$ and bypass flow rate $q$. It should be especially mentioned here, that it is not necessary and above all not possible to simulate of full $1 \mathrm{MW}$ power, resulting from the $600 \mathrm{MeV}$ proton beam, which will be used for MEGAPIE target. Our pre-test estimations show that the heating power range, that is necessary to achieve sufficient heat flux, is between $1 \mathrm{~kW}$ and $10 \mathrm{~kW}$. For this reason, we have named our investigation the KILOwatt PIlot Experiment (KILOPIE).

\section{SET-UP OF KILOPIE IRT EXPERIMENT SERIES}

\subsection{General set-up of KILOPIE IRT experiments}

We have used the Two-Dimensional and Dynamic Infrared Thermography (2DD-IRT) method, for the estimation of the flow characteristics in a convection boundary layer before with good results [2], [5]. The most interesting aspects of IRT are non-contact field temperature measurement techniques and high geometrical- $(1.25 \mathrm{mrad})$ and time- $(20 \mathrm{~Hz})$ resolution. These advantageous features have been fully used for the visualizations produced by 2DD-IRT method.

In general, from the thermo-hydraulics point of view the theoretical model and methodology used is presented in sections 4, 5 and 6. For the determination of local heat transfer coefficient HTC four physical values (see Eq.4.11) have to be determined by measurements: local heat flux $\left(q^{*}\right)$ from heating foil, coolant bulk temperature $\left(T_{b u l k}\right)$, outside temperature of the surface of the heating foil $\left(T_{\text {out }, \text { foil }}\right)$ and the thermal resistance $\left(R_{t h}\right)$ of the whole multilayer specimensensor wall. These measurements define the instrumentation and the set-up of the experiment.

The entire set-up with all the instrumentation of the IRT KILOPIE experiment is shown in Fig.2. The interface to LM loop and to the specimen sensor dish so-called "three flanged vessel", representing the lower part of the MEGAPIE target has been fabricated from stainless steel. The smallest lower flange is fixing the intrinsic specimen sensor dish, i.e. a simulation of the target window the proton beam loaded area. The second middle flange represents the interface to the

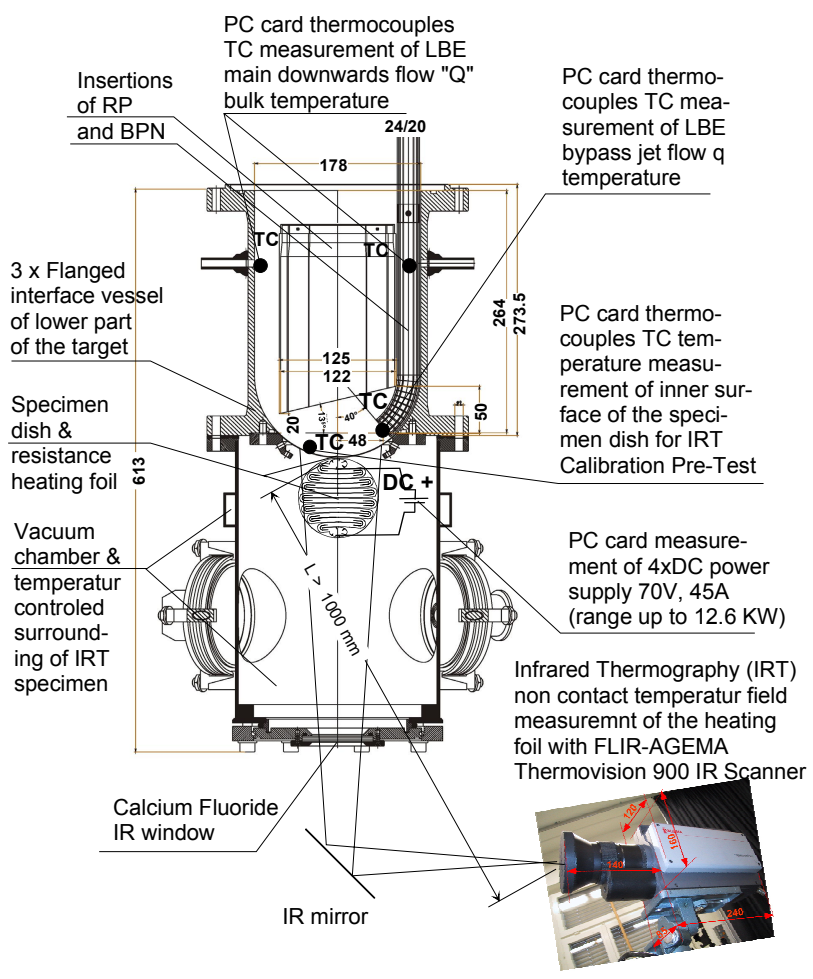

Figure 2: IRT KILOPIE experimental set-up and instrumentation.

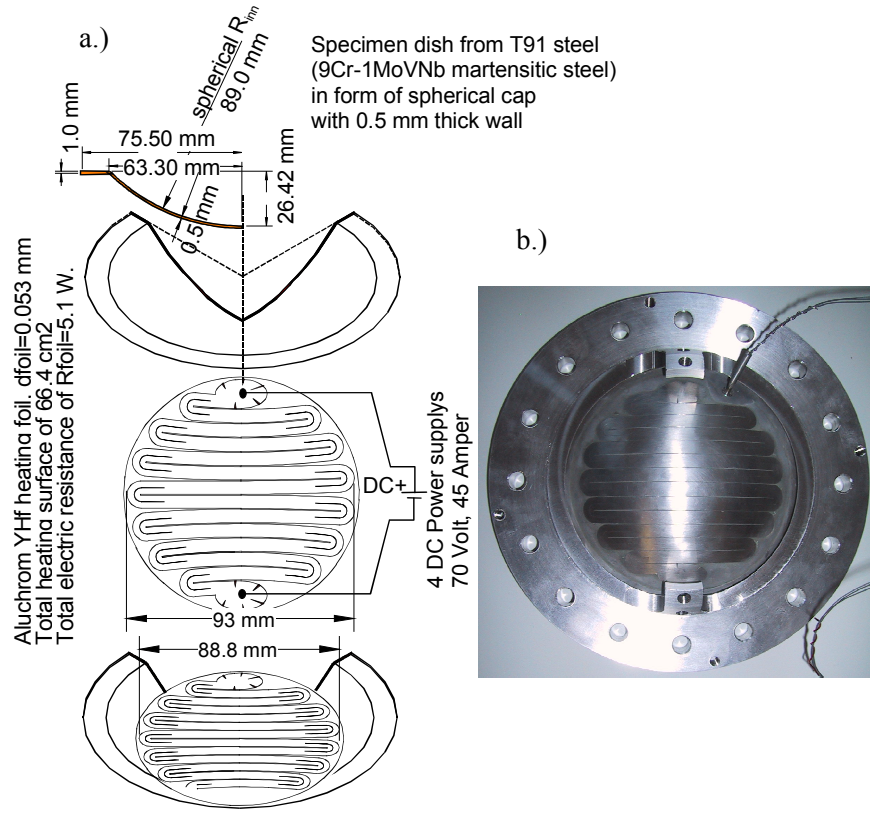

Figure 3:IRT KILOPIE specimen sensor dish with resistance heating foil:

a.) Structure of multilayer specimen sensor dish.

b.) Photography of the specimen sensor dish with lower flange and thermocouples placed on inner surface of the dish. 
so-called "vacuum chamber", a device for realization of adiabatic boundary conditions for the IRT measured specimen. Controlling the surrounding ambient temperature, i.e. walls of the vacuum chamber, reduces the radiation loss. And finally the upper flange is serving as direct connection to different LM loops used.

The specimen sensor dish, see Fig.3, consists of a steel dish in the form of a $0.5 \mathrm{~mm}$ thin spherical cap manufactured from real target window material, T91 steel $(9 \mathrm{Cr}-1 \mathrm{MoVNb})$ and a resistance heating foil glued on the outer surface of the dish with a special procedure. The IRT scanner FLIR-AGEMA Thermovision 900 measures the outer temperature field of the specimen from the distance of approx. $1000 \mathrm{~mm}$ through a calcium fluoride crystal glass at the lowest part of the vacuum chamber. For safety reasons the IRT scanner is placed $90^{\circ}$ out of the axis and takes measurement from the heat radiation reflected by a high quality polished IR mirror. It is necessary to save the expensive IRT scanner in the hypothetical accident case of the break of both the specimen dish and calcium fluoride IR window.

\subsection{Preparation of the sensor dish resistance heating}

A suitable heating mechanism for the window was chosen in view of optimizing the conditions for IRT measurements and experimental goals. Thus the following measures and design choices were taken:

- To heat the target window's mock-up by volumetric resistance heating within the thin metallic foil glued to the outer surface of specimen. Such heating is well suited for use with IRT because heat reflection from the window surface is avoided. In the converse case of the external heating, an outside heat source would be reflected in the nonblackbody surface of the window's mock-up and strongly disturbs the IRT measurements.

- To be able to realize in the temperature range $180-400^{\circ} \mathrm{C}$, the desirable, constant and homogenous distributed heat flux value $q^{*}$ over the well known heating area applied to the target window's mock-up.

- To screen the target window's mock-up with a surrounding anti-reflection vacuum chamber so as to avoid the effects of ambient heat sources at the sides (e.g. electric instruments/components or walking persons).

- To enhance the emissivity $\varepsilon$ of the window surface (i.e. heating foil) and make it uniform. For example to this end the outer surface of the heating foil has been covered with special few micrometers thin alumina coating spray.

- To perform an exact calibration of emissivity $\varepsilon$ for the window's mock-up surface after each change of experimental configurations. The so-called emissivity chart technique [6] has been used, which gives an individual value to the emissivity $\varepsilon$ of each measurement pixel (see section 5.1).

- To perform an exact calibration of thermal resistance $R_{t h}$ for the window's mock-up wall, which gives an individual value to thermal resistance $R_{t h}$ of each measurement pixel (see section 5.2).

An important criterion for the material of the resistance heating foil is a thermal independency of electrical resistivity. It is a big advantage to have the constant heat flux during the different cooling over whole area of observed temperature changes on the surface of the foil. The temperature distribution on the foil surface can differ in the range from LBE bulk temperature, e.g. $180^{\circ} \mathrm{C}$, up to approx. $400^{\circ} \mathrm{C}$, as in case of low efficiency cooling. Finally we have chosen the Aluchrom YHf ferritic steel ( $\mathrm{CrAl} 205$ ), with constant resistivity of $1.41 \mathrm{E}-6 \Omega \cdot \mathrm{m}$ in the temperature range $200-400^{\circ} \mathrm{C}$.

The applied heat flux $q^{*}$, as experiment's parameter, should vary between $5-125 \mathrm{~W} / \mathrm{cm}^{2}$. For example for one series of experiment we have used $5.1 \Omega$ heater, consequently the suitable power supply has to cover the range from $332 \mathrm{~W}$ for the desired lowest heat flux of $5 \mathrm{~W} / \mathrm{cm}^{2}$ up to $8300 \mathrm{~W}$ for the desired heat flux of $125 \mathrm{~W} / \mathrm{cm}^{2}$. The gluing procedure to the steel dish has been done in two steps. The first one consists with covering the steel surface with approx. $50 \mu m$ thick ceramic layer as electric insulation. The second step is the application of the steel heating foil with special epoxy glue. The thickness of this epoxy layer should bee approx. $5 \mu \mathrm{m}$. In reality layers are not uniform and varied up to $100 \mu \mathrm{m}$.

Most important for the determination of the local convection heat transfer coefficient $H T C$ is precise determination of the heat flux. The meander tailoring of the heating foil with constant thickness, e.g. as has been used in one experiment series $0.053 \mathrm{~mm}$, should guarantee the homogenous distribution of the total power generated in the foil over the whole heated specimen surface. To give the reader more feeling for the mentioned example the electrical resistance of the heating foil is known $R_{\text {foil }}=5.1 \Omega$, as well as the area of the heated surface of the specimen spherical cap laying under the foil $A_{\text {cap }}=66.39 \mathrm{~cm}^{2}$. The desired heat fluxes, e.g. $q^{*}=5,10$ and $15 \mathrm{~W} / \mathrm{cm}^{2}$, allow calculate according the Eq. 3.1 the need total power: the corresponding values are $P_{\text {total }}=332 \mathrm{~W}, 664 \mathrm{~W}$ and $996 \mathrm{~W}$. According well known Ohm's Law, Eq.3.2 we can asses the desired current values that shoud be realised through the DC power supply units.

"The three LM (LBE) loops were used for different series of experiments:

1. THEADES loop of the KALLA laboratory at Forschungszentrum Karlsruhe (FZK), Germany

2. Double pumped circuits PSI- LBE loop at Paul Scherrer Institute (PSI), Switzerland

3. MEGAPIE Integrated Test (MIT) target at Paul Scherrer Institute (PSI), Switzerland 
$P_{\text {total }}=q^{*} \cdot A_{\text {cap }}$

$P_{\text {total }}=I^{2} \cdot R_{\text {foil }}=U^{2} / R_{\text {foil }}$

where: $I$ is DC current and $U$ is DC voltage

The corresponding DC current and voltage values are: for $q^{*}=5 \mathrm{~W} / \mathrm{cm}^{2} ; 8 \mathrm{~A}, 41 \mathrm{~V}$, for $q^{*}=10 \mathrm{~W} / \mathrm{cm}^{2} ; 11.4 \mathrm{~A}, 58 \mathrm{~V}$, and for $q^{*}=15 \mathrm{~W} / \mathrm{cm}^{2} ; 14 \mathrm{~A}, 71.4 \mathrm{~V}$. The DC current values have been simultaneously measured and recorded with all other TC and IRT measurements.

\subsection{Instrumentation of KILOPIE IRT experiments}

As shown in Fig. 3, the general instrumentation used in these experiments consists of two temperature measurement devices: the IRT equipment and the thermocouples TC equipment.

The precise control that is needed to keep the pumping parameters, main flow rate $Q$ and by-pass flow rate $q$, of the loop at their prescribed values, to hold the heating DC current constant and to maintain the security of the whole facility, will not be described in this paper.

The IRT equipment of PSI (FLIR-AGEMA THV900 SWTE) is a non-contact measurement system, which allows 2D thermograms in the infrared short wave spectrum range (2-5.6 micrometer) to be registered. The IR- thermograms can be collected as single frames or as sequences. The time resolution for on-line recording by the IR-scanner and controller vary from $20 \mathrm{~Hz}$ for frames consisting of 128 lines at 204 pixels up to $3500 \mathrm{~Hz}$ for single lines of 204 pixels. The spatial resolution depends on the optic lens of the IR-scanner. In our IRT measurements a $10^{\circ}$-teleobjective and $20^{\circ}$ standard lenses with a spatial resolution of 1.25 and $2.5 \mathrm{mrad}$ respectively have been used. For a more detailed technical specification of FLIR-AGEMA THV900 SWTE, see User's Manual [6]

The data acquisition system for the TCs consists of a PC and Keithley or National Instruments cards. Very thin thermocouples (diameter $0.25 \mathrm{~mm}$ ) of the "K" type, produced and calibrated at PSI guaranteed response times shorter than $20 \mathrm{~ms}$ and reached the high accuracy of $\pm 0.2^{\circ} \mathrm{C}$.

The data sets from the IRT and from the TC measurement must be recorded simultaneously. In order to achieve this synchronization, a special trigger unit was built to start each measurement or simply the network based time synchronization for all involved computers has been used.

\section{THEORETICAL MODEL FOR EVALUATION OF THE DATA MEASURED IN THE KILOPIE IRT EXPERIMENT}

In the convection heat transfer problem with predominate one dimensional heat flux trough a steel wall, the local heat flux q* may be expressed as

$$
q^{*}=H T C \cdot\left(T_{\text {inn,steel }}-T_{\text {bulk }}\right)
$$

where: $q^{*}$ is local heat flux, HTC is local convection heat transfer coefficient, $T_{i n n, s t e e l}$ is temperature on the inner flow contact surface of the steel wall and $T_{b u l k}$ is bulk temperature of the LM coolant LBE flow medium.

The set-up of IRT KILOPIE Experiment is based on measurements of components from Eq.4.1 direct used for calculation of $H T C$. The LBE bulk temperature $T_{b u l k}$ will be directly measured by TC measurement (see section 3, Fig.2). The values of desirable heat flux $q^{*}$ are determined according Eq. 3.1 and Eq. 3.2, by DC current measurement.

A little more complicated will be the determination of the inner wall temperature, $T_{i n n, \text { steel }}$. Because IRT can give only the values of the temperature $T_{\text {outffoil }}$ of the outer surface of the heating foil, the problem arises of estimating the difference between this temperature and the temperature $T_{\text {inn,steel }}$ at the inner surface of the window. This aspect is

discussed below. Knowledge of the behavior of $\Delta T_{\text {wall }}=T_{\text {out.foil }}-T_{\text {inn,steel }}$ during the IRT KILOPIE experiments is essential for the interpretation of the IRT measurements.

As mentioned in section 3 the specimen is surrounded by a vacuum chamber. Therefore only the radiation loss of the energy trough the outer surface of the heating foil can take place. The temperature $T_{a m b}$ of the wall of the vacuum chamber is controlled during the measurements by a special water channel; a difference between the ambient temperature $T_{a m b}$ and $T_{\text {out.foil }}$ is known and can be kept constant and as small as necessary for IRT. In such conditions the energy radiation flux $q^{*}{ }_{\text {rad }}$ can be neglected, especially in comparison with the convective heat flux $q^{*}$ conv on the inner surface of the specimen dish. It therefore can be assumed that the average heat flux on the outer surface is zero. In conclusion, quasi adiabatic boundary conditions can be assumed on the outer surface of the heating foil.As the wall thickness $\delta=0.5 \mathrm{~mm}$ of the dish is a small fraction of its diameter $D=178 \mathrm{~mm}$, it allows assume a 1-D conduction heat flux in the x-direction. This model can be applied to each small local area of the specimen wall because the predominant heat flux is normal to the surface of heated spherical cap. The main aspects of the model are shown in Fig.4. The conversion from electrical to thermal energy will take place only within the thickness of the heating foil layer of the multilayer wall of the specimen 


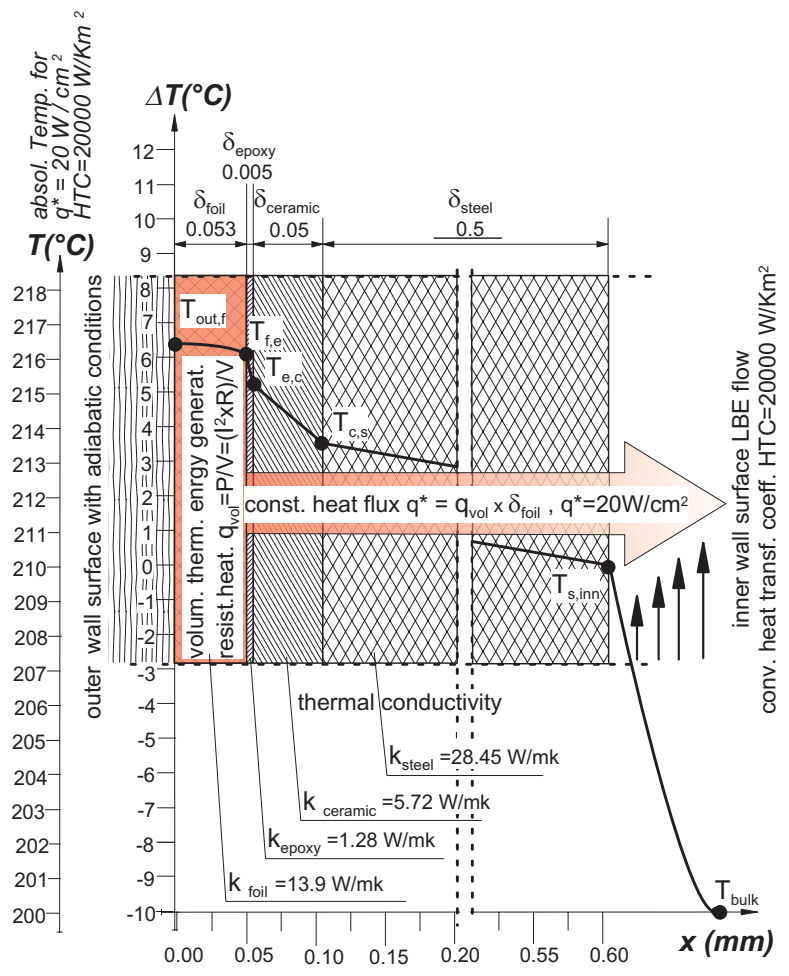

Figure 4: IRT KILOPIE;1-D heat flux model for the multilayer wall of specimen sensor dish with heating foil glued to the T91 steel. An example for approximately numerical values of temperature distribution within the wall with material bulk conductivities from literature and ideal thermal contacts between in the layers. For this simplified calculation following input values are assumed:

$T_{\text {bulk }}=200^{\circ} \mathrm{C}$, heat flux $q^{*}=20 \mathrm{~W} / \mathrm{cm}^{2}$ and $H T C=20000 \mathrm{~W} / \mathrm{Km}^{2}$. dish and at a local volumetric rate denoted by $q_{v o l}$. The consequence of this heat generation is that the heat flux is no longer independent of $x$, and this must be considered in the model calculations.

Summarized: the small ratio of the thickness $\delta$ to the diameter $D$ of the spherical cap dish and as inward convective heat flux perpendicular to the wall predominates, the simple steady-state 1-D model Fig.4 with uniform energy generation per unit volume in the first heating foil layer of composite wall $q_{v o l}=$ constant and constant thermal conductivities $k_{\text {foil }}, k_{\text {epox }}, k_{c e r}, k_{\text {steel }}$, through all layers can be used for the plane wall.

The appropriate heat equation for the foil layer is then

$$
\frac{d^{2} T(x)}{d x^{2}}+\frac{q_{v o l}}{k_{\text {foil }}}=0
$$

where: $k_{f o i l}$ - is thermal conductivity of the heating steel foil, $q_{v o l}$-is local energy generation per unit volume.

The general solution of Eq.4.2 is

$$
T(x)=-\frac{q_{v o l}}{2 k_{f o i l}} \cdot x^{2}+C_{1} \cdot x+C_{2}
$$

The constant of integration $C_{l}=0$ results directly from the assumption of adiabatic boundary conditions $(d T / d x)_{(x=0)}=0$ on the outer surface of the mockup, as discussed above. The constant of integration $C_{2}=T_{\text {out.foil }}$ is directly evaluated from the boundary conditions $T_{(x=0)}=T_{\text {out.foil }}$, which are known from the IRT measurement. Consequently the temperature distribution is given by Eq. 4.4.

$$
T(x)=-\frac{q_{v o l} \cdot x^{2}}{2 \cdot k_{\text {foil }}}+T_{\text {out.foil }}
$$

Inserting $x=\delta_{\text {foil }}$ into Eq.4.4, the temperature $T_{\left(x=\delta_{\text {foil }}\right)}=T_{\text {foil.epox }}$ on the inner surface of the wall is given by Eq.4.5.

$$
T_{\text {foil }, \text { epox }}=-\frac{q_{v o l} \cdot \delta_{\text {foil }}^{2}}{2 \cdot k_{\text {foil }}}+T_{\text {out.foil }}=-\frac{q^{*} \cdot \delta_{\text {foil }}}{2 \cdot k_{\text {foil }}}+T_{\text {out.foil }}
$$

where: $q^{*}=\delta_{\text {foil }} \cdot q_{v o l}$ is the local heat flux, other symbols see Eq.4.2 and text. The desired value for $\Delta T_{\text {foil }}=T_{\text {out }, \text { foil }} T_{\text {foil,epox }}$ is then given by Eq.4.6.

$$
\Delta T_{\text {foil }}=T_{\text {out.foil }}-T_{\text {foil }, \text { epox }}=\frac{q^{*} \cdot \delta_{\text {foil }}}{2 \cdot k_{\text {foil }}}
$$

For the heating foil layer we can express the local heat flux in dependency of temperature. Because the heat flux is constant through the specimen wall, and for another layers, i.e. epoxy (glue), ceramic (electrical insulation) and dish steel, linear conduction takes place, the equations for heat flux for each layer expressed in dependency with temperature are for the epoxy layer, the ceramic layer and the shell dish wall layer(eq 4.7),

$$
q^{*}=\frac{T_{\text {out }, \text { foil }}-T_{\text {foil }, \text { epox }}}{\frac{\delta_{\text {foil }}}{2 k_{\text {foil }}}}=\frac{T_{\text {foil }, \text { epox }}-T_{\text {epox }, \text { cer }}}{\frac{\delta_{\text {epox }}}{k_{\text {epox }}}}=\frac{T_{\text {epox }, \text { cer }}-T_{c e r, \text { steel }}}{\frac{\delta_{c e r}}{k_{c e r}}}=\frac{T_{\text {epox }, \text { cer }}-T_{c e r, \text { steel }}}{\frac{\delta_{c e r}}{k_{c e r}}} .
$$

Finally the same heat flux $q^{*}$ is going by convection to LBE bulk flow from the inner surface of the steel wall of the 
specimen sensor dish, as it has been mentioned at the beginning of this chapter, see Eq. 4.1.

From Eq.4.7 we can reduce all temperatures inside of the specimen wall, therefore

$$
q^{*}=\frac{T_{\text {out }, \text { foil }}-T_{\text {inn,steel }}}{\frac{\delta_{\text {foil }}}{2 k_{\text {foil }}}+\frac{\delta_{\text {epox }}}{k_{\text {epox }}}+\frac{\delta_{\text {cer }}}{k_{\text {cer }}}+\frac{\delta_{\text {steel }}}{k_{\text {steel }}}}
$$

In this place it should be mentioned that the whole expression in denominator of Eq. 4.8 represent a thermal resistance of the multilayer wall for the simplified one-dimensional ideal conduction heat transfer model.

In the fact between the layers some additional so called a thermal contact resistance $R_{t h, c}$ can exist (e.g. see [7], Chapter 3.1.4 and corresponding references). Therefore the total thermal resistance $R_{t h}$ of the multilayer wall can be expressed as in Eq. 4.9.

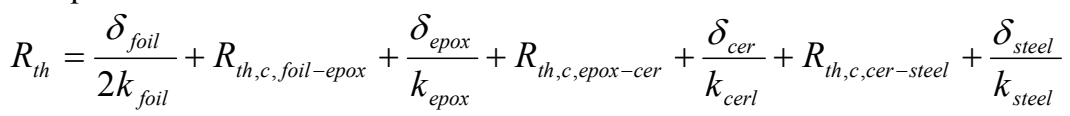

Since the values of the thermal contact resistances depend strongly on the manufacturing conditions and generally are unknown, the total thermal resistance $R_{t h}$ cannot be calculated only from known thicknesses and bulk conductivities of materials and has to be determined experimentally in separate calibration tests.

Eq.4.8 can be converted to the simple Eq. 4.10, which allows calculating the unknown temperature on the inner surface of the steel dish.

$$
T_{\text {inn,steel }}=T_{\text {out }, \text { foil }}-q^{*} \cdot R_{\text {th }}
$$

Finally, putting Eq.4.10 into Eq.4.1 we obtain Eq.4.11, which allows the determination of the local convection heat transfer coefficient $H T C$

$$
H T C=\frac{q^{*}}{T_{\text {out }, \text { foil }}-q^{*} \cdot R_{\text {th }}-T_{\text {bulk }}}
$$

expressed with values measured during the KILOPIE IRT Experiment.

\section{CALIBRATION TESTS}

\subsection{Emissivity calibration tests}

The preparations described below are a part of the intense efforts that were made to measure the absolute temperature $T_{\text {out.foil }}$ on the outer surface of the foil glued to the specimen dish. The difference to the absolute value $T_{\text {bulk }}$ of the LBE bulk temperature is essential for the evaluation of the results.

The use of IR-thermography to derive absolute values of temperature requires the value of the emissivity $\varepsilon$, a radiative property of the surface, to be known. The emissive power $E$ of the surface, which is the quantity directly measured in the IR-scanning, is less than the corresponding quantity $E_{b b}$ of blackbody radiation at the same temperature and is given by the Stefan-Boltzmann law,Eq.5.1

$$
E=\varepsilon \cdot \sigma \cdot T^{4}
$$

where: $\varepsilon$ - emissivity; radiative property of the surface values in the range (0-1), $\sigma$-is Stefan-Boltzman constant; $\left(\sigma=5.67 \mathrm{E}-8 \mathrm{~W} / \mathrm{m}^{2} \mathrm{~K}^{4}\right)$, and $T$-absolute temperature in $K$.

For each measurement pixel, the emissivity can be expressed as a function of the spectral range $\lambda$, the temperature $\mathrm{T}$, the direction of radiation $\phi$ (i.e. the angle between the view line and the normal to the radiating surface) and the roughness $R_{a}$ :

$$
\varepsilon=F\left(\lambda, T, \phi, R_{a}\right)
$$

Because the spectral $\lambda$-dependency of the IR scanner-detector is generally constant within the spectral range of IRT equipment used (in our case $\lambda=2-5.6 \mu \mathrm{m}$ ), only the influence of the remaining three variables has to be considered for our experiments. These are:

- the cleanliness/roughness $(R a)$ of the surface ; for enhancement of the emissivity for same experiment series the surface was treated on a mechanical or chemical way (fine sand blasting techniques, alumina powder spraying, covering with selenium oxide)

- the position or i.e. the direction view angle $(\phi)$ of the hemispherical surface relative to the IR scanner, and

- the temperature of the window surface $(T)$.

In order to find these dependencies, special calibration tests were performed "in situ" on the experiment stand. The 
calibration tests also serve to determine directional spectral emissivity charts [6] for precisely pixel-manner correction of IRT thermograms. The stable and uniform temperature fields have been recorded with IRT and TC equipment, using the $\mathrm{TC}$ values as reference.

During the calibration test the outer surface of the sensor dish has been heated passively by the hot LM LBE, i.e. without heat flux generated by electrical DC current and therefore in steady state, without temperature gradient within the wall thickness . For this passively heating, only the LBE main flow $Q$ with constant $T_{b u l k}$ temperature has been used. The criterion for achieving of steady state $T_{b u l k}=T_{\text {inn,steel }}$ has been controlled by thermocouples (TCs). The TCs used to measure the reference temperature $T_{i n n, \text { steel }}$ on the dish and to control the uniformity of the temperature field are placed on the inner steel surface of the dish at few points along one radius. In this way a uniform temperature field $T_{\text {out foil }}=T_{\text {inn,steel }}=T_{\text {bulk }}$ was established on the whole outer, quasi adiabatic (vacuum insulated) surface of the sensor dish. Few steps of temperature within the expected range from $180^{\circ} \mathrm{C}$ up to $300^{\circ} \mathrm{C}$ have been measured in order to obtain the temperature dependence of the emissivity. The resulting emissivities charts obtain with Erika software [6] were stored for later correction of all IRT measurements. The IRT measurements of outer surface, i.e. foil layer, have been done from precisely the same position as later in the main experiment; therefore aligning of all IRT scanner records to emissivity chart is assured. According to expectations the results show, that the normal spectral emissivity of T91 steel polished surface of approx. $\varepsilon=0.14$ has been enhanced to $\varepsilon=0.41$ an alumina sprayed surface, to $\varepsilon=0.55$ for a specially specified low energy fine sand blasted steel surface and enhanced to $\varepsilon=0.51$ for the thin layer of selenium oxide surface. A small and for correction of single $Q / q$ LBE flow cases meaningless temperature dependency of normal spectral emissivity has been found for alumina sprayed surface only. The normal spectral emissivity of sand blasted and chemical treated surface (thin layer of selenium oxide) was constant within tested $180-250^{\circ} \mathrm{C}$ temperature range.

\subsection{Thermal resistance calibration tests}

Because of the multilayer construction of the specimen sensor dish with not enough precisely known heat conductivity of the single layers and unknown contact thermal resistance between layers the total thermal resistance $R_{t h}$ of the specimen sensor dish wall has to be determined experimentally (see section 4, Eq.4.9). This $R_{t h}$ value is necessary for the calculation of inner surface temperature from the IRT measured outer surface temperature of the specimen sensor dish according Eq.4.10 and subsequently for the determination of $H T C$ according Eq.4.11.

Additionally non homogeneous distribution field of the thickness of the glue layer, caused by the hand made technology, complicates task of HTC determination. With other words, because this deficiency with thickness of glue layers, for the determination of $H T C$ distribution field, is necessary to measure the distribution field of the total thermal resistance $R_{t h}$. of the sensor dish multilayer wall. This task is solved by applying a triple procedure:

\#1. Measurement of temperature conductivity using standard Laser Flash Analysis (LFA) and subsequently calculation of the heat conductivity and finally the thermal resistance $R_{t h}$. The LFA measurements have been done for total thickness of multilayer wall specimen (small round $10 \mathrm{~mm}$ diameter plane plate), cutt from the wall identically manufactured as the sensor dish.

\#2. In presence of heat flux $q^{*}$ measurement of the distribution of the local thermal resistance $R_{t h}$ for the total thickness of the multilayer wall in a special calibration tests performed on the whole sensor dish using so called "mixing ice water" procedure.

\#3. Measurement of the distribution of the local thermal resistance $R_{t h}$ for the total thickness of multilayer wall in special calibration test performed "in situ" on the experiment stand on the whole sensor dish using the LM LBE flow created by main pump.

The homogenous and constant heat flux $q^{*}$ applied in both last procedures $\# 2$ and $\# 3$ has been created within heating foil layer of the specimen sensor dish. Also both procedures the \#2 with mixed ice water and the \#3 with "in situ" LBE flow condition should create on the inner surface the special conditions with the constant and homogeny heat convection field of local heat transfer coefficient. The absolute value of this special HTC has not to bee known. For the known homogenous heat flux $q^{*}$ (as described in section 3.2) and known temperature of the inner surface $T_{\text {inn }}^{\text {Rtseel }}$ measured with TCs (for this special HTC the $T_{\text {inn,steel }}^{\text {Rtest }}=$ const.) and simultaneously IRT measurement of temperature of outer surface of the heating foil $T_{\text {out } \text { foil }}^{\text {Rt }}$ make possible to determine the distribution of local $R_{t h}$ according Eq.5.3.

$$
R_{\text {th }}=\left(T_{\text {out }, \text { foil }}^{\text {R tel }}-T_{\text {inn,steel }}^{\text {Rtest }}\right) / q^{*}
$$

The procedures \#2 and \#3 are serving for the creating of the total thermal resistance chart, similar to the emissivity chart and consequently, for each heat flux used as parameter, the thermal resistance correction temperature chart. The procedure \#1 is serving for the additional controlling of the total thermal resistance chart. 


\section{REALIZATION AND PROGRESSION OF THE KILOPIE IRT MEASUREMENTS}

As it was mentioned in the scope of KILOPIE the different experimental series have been performed KILOPIE-1 [8], KILOPIE-2 PRE [9] and KILOPIE MIT [10]. Each series was an iteration step for better understanding of this construction type of LM cooling and has led to improvements of the measurement procedure. For example only for one so called reference nozzle shape with the rectangular $20 \mathrm{~mm} \times 10 \mathrm{~mm}$ shape for the by-pass jet flow $q$, the long way of the optimum solution finding has been done, they were together 84 different $Q / q$ flow cases where the IRT measurements have been performed.

Other parameters are heat flux and bulk temperature of LM (LBE): e.g. in KILOPIE-1 series were: the LBE bulk temperature $T_{\text {bulk }}=180^{\circ} \mathrm{C}$ a equal for both $q$ and $Q$ flows, the foil heating heat fluxes $q^{*}=5$ and $10 \mathrm{~W} / \mathrm{cm}^{2}$.

All additional parameters have multiplied the number of IRT measurements and enlarged our experience connected with using this method. Finally we have worked out a general similar procedure which we have followed for all geometrical configurations of the IRT KILOPIE experiments and for the $\boldsymbol{Q} / \boldsymbol{q}$ flow pairs from the experiments flow matrices.

- The inner geometrical configuration of the flow and interface 3-flange vessel with: specimen sensor dish, power and TC signals measurements cabling and vacuum chamber have been mounted to the LM LBE target/loop.

- The target/loop has been preheated and LBE filled

- The vacuum and tempering of walls of the vacuum chamber have been realized (as optimal IRT measurements conditions for outer surface in quasi adiabatic conditions).

- The common start of IRT, TC and DC measurements recording (simultaneously started with the triggering device).

- The separate conditions for "in situ" emissivity (only $\boldsymbol{Q}$ flow in absence of heat flux $\boldsymbol{q}^{*}$ ) and thermal resistance (only $-\boldsymbol{Q}$ flow in presence of heat flux $\boldsymbol{q}^{*}$ ) calibration tests have been established and pre-tests have been performed.

- The settings of steady state $\boldsymbol{Q} / \boldsymbol{q}$ flows and equalization of the bulk temperatures $\boldsymbol{T}_{\boldsymbol{b u l k}}$ for $\boldsymbol{q}$ and $\boldsymbol{Q}$ flows have been realized.

- Continuation recording phase $\boldsymbol{Q}+\boldsymbol{q}$ flows in absence of heat flux, $\mathrm{q}^{*}=\mathbf{0}$, no current on heating foil (optional, duration e.g. ca.60 sec.)

- Constant heat flux $q^{*}$ on; Switching on of proper DC current/voltage value for the heating foil

- Continuation recording phase $\boldsymbol{Q}+\boldsymbol{q}$ flows in presence of desired heat flux $\boldsymbol{q}^{*}$, (duration e.g. ca. $60 \mathrm{sec}$.)

- The by-pass LM jet flow $\boldsymbol{q}$ has been stopped.

- Continuation recording phase only $\boldsymbol{Q}$ flow in presence of heat flux $\boldsymbol{q}^{*}$, (duration e.g. ca.60 sec.)

- Stop of heat flux $\boldsymbol{q}^{*}=\mathbf{0}$, heating foil current switched off

- Continuation recording phase only $\boldsymbol{Q}$ flow in absence of heat flux $\boldsymbol{q}^{*}=\mathbf{0}$, (duration e.g. ca.60 sec.)

- Stop of data recording.

We will accentuated, that all members of denominator of Eq.4.11 can be obtain as IRT temperature fields during above described IRT measurement procedures. Therefore inclusive separate calibration tests Eq.5.3 we can write Eq.6.1

HTC $=\frac{q^{*}}{\left(T_{\text {out }, \text { foil }}-T_{\text {bulk }}\right)-\left(T_{\text {out }, \text { foil }}^{\text {R tost }}-T_{\text {inn }, \text { steel }}^{\text {test }}\right)}$

which shows, that during IRT data evaluation the local heat transfer coefficient can bee constructed from a one final difference temperature field, which can be obtain from two temperature difference fields as grouped in denominator of Eq.6.1. The final arithmetic to achieving the local HTC values building the two-dimensional (2D) $H T C$ chart is multiplication a reciprocals of this final temperature difference field by constant value of applied heat flux $q *$.

\section{SOME RESULTS OF IRT KILOPIE EXPERIMENT SERIES}

For the reader who is interested in quantitative IR thermography we want to present not only the final results of the thermo-hydraulics experiment i.e. HTC values for LM LBE cooled hemispherical steel target window, but also the intermediate steps of processing result of the IRT measurement procedure steps as e.g. the emissivity- and thermal resistance -charts.

From the abundance of investigated cases we have chosen as a representative illustration case MP10mixBP02V75, from the KILOPIE-2 experiment series with the reference geometrical configuration, i.e. the by-pass outlet is tangential 
to the hemispherical window's shell and the nozzle has $10 \times 20 \mathrm{~mm}$ rectangular shape.

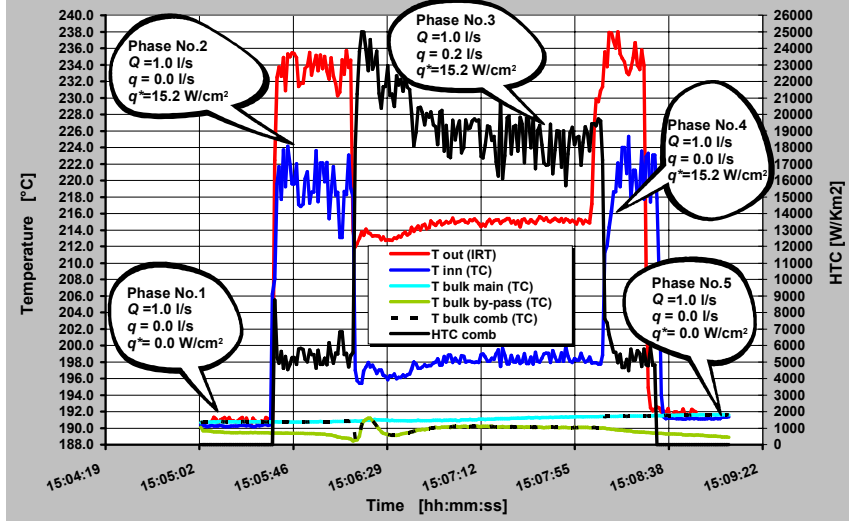

Figure 5: Time history of flow case MP10mixBP02V75. Data for the central point of the specimen sensor dish, values of $T_{\text {out }}, T_{i n n}$, different $T_{\text {bulk }}$ and $H T C$

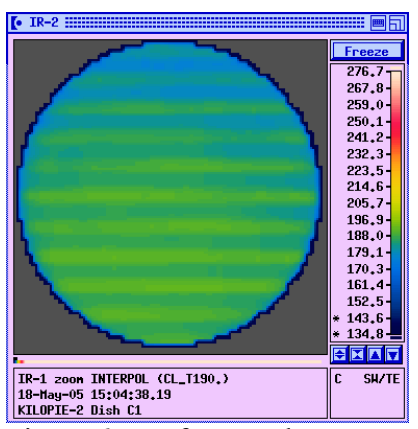

Figure 6a: Reference thermogram for emissivity chart at equalized temperature $T_{\text {out }, \text { foil }}=$ $=T_{\text {inn,steel }}=T_{\text {bulk }}=190.2^{\circ} \mathrm{C}$

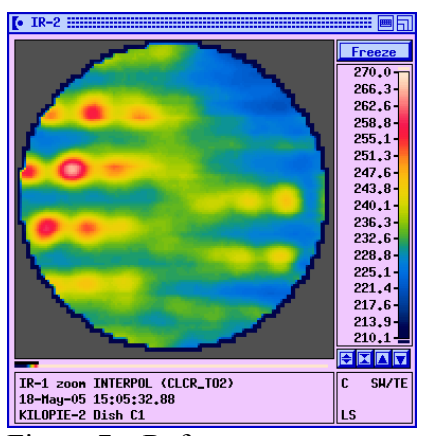

Figure 7a: Reference

thermogram $T_{\text {out, foil }}^{\text {Rtest }}$ for

thermal resistance calibration by presence of the heat flux $q^{*}=15.2 \mathrm{~W} / \mathrm{cm}^{2}$ correcting showing hot spots caused by glue-clumps

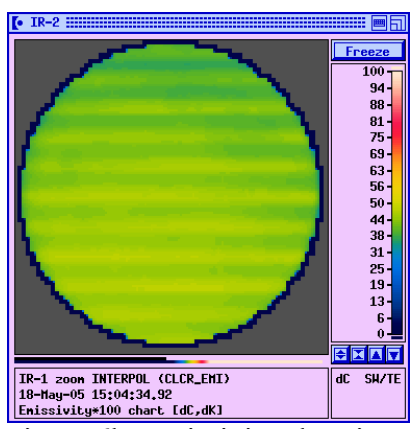

Figure 6b: Emissivity chart in $[\%]$ for the specimen sensor dish used in case MP10mixBP02V75

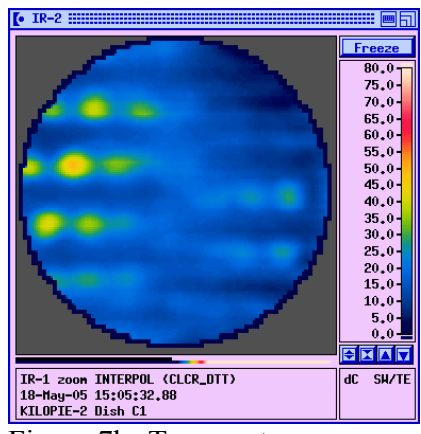

Figure $7 \mathrm{~b}$ : Temperature difference field from Eq.6.1 $\left(T_{\text {out }, \text { foil }}^{R \text { tost }}-T_{\text {inn, steel }}^{R \text { test }}\right)=q * R_{\text {th }}$ by the heat flux $q^{*}=15.2 \mathrm{~W} / \mathrm{cm}^{2}$ which is correcting the gluing deficiencies resulting in non uniform thermal resistance
The time histories of this case for temperatures at the central point of the sensor dish are shown in Fig.5. The case is characterized by 5 sequential phases: phase \#1 with only main pump forced flow of $\boldsymbol{Q}=1.0 \mathrm{l} / \mathrm{s}$ and $T_{b u k l}$ $=190.2^{\circ} \mathrm{C}$, phase \#2 additionally with the heat flux $\boldsymbol{q}^{*}=15.2 \mathrm{~W} / \mathrm{cm}^{2}$ applied, phase \#3 the by-pass pump forced flow $\boldsymbol{q}=0.2 \mathrm{l} / \mathrm{s}$ of the jet cooling additionally switched on, phase \#4 the by-pass jet flow switched off $(\boldsymbol{q}=0.0 \mathrm{l} / \mathrm{s})$ and finally phase \#5 the DC current of the heating switched off $\left(\boldsymbol{q} *=0 \mathrm{~W} / \mathrm{cm}^{2}\right)$.

Fig. 5 shows: the IRT measured outer sensor dish surface temperature $T_{\text {out foil }}$, the TC measured inner sensor dish surface temperature $T_{\text {inn,steel }}$, the LBE bulk temperatures $T_{\text {bulk }}$ of the main- and by-pass-flow, and the combination of both, which is valid for the central point of the sensor dish. Also the HTC values calculated according to Eq.4.1 are plotted.

In order to obtain true temperatures from the IRT measurements we first have to perform correction of primary used constant uniform emissivity. The results of emissivity correction procedure according section 5.1 are shown in Fig.6, where the $100 \mathrm{~mm}$ diameter FOV of sensor dish central axial projection is represented. We used the phase \#1 to do it, with thermal conditions $T_{\text {out,foil }}=T_{\text {inn,steel }}=T_{\text {bulk }}=190.2^{\circ} \mathrm{C}$, which comply condition of emissivity calibration test. Fig.6a shows the non corrected thermogram, where the pattern of the heater foil strips and gaps can be seen, because of the rest transparency of sprayed alumina powder. Fig.6b shows the emissivity chart obtain with Erika software [6], which is consequently used for correction of all IRT records of this case. The real directional spectral emissivities of the sensor dish surface vary in range 0.37 0.48 .

In a second step we have to determine the correction values for the inhomogeneity in the thermal resistance according to procedure given in section 5.2. The manually applied electrical insulation and glue layers vary in thickness and cause irregular distributed hot spots on the outer surface of the sensor dish. Fig. 7a shows emissivity corrected surface temperature $T_{\text {out, foil }}^{R \text { test }}$ at the constant heat flux $q^{*}=15.2 \mathrm{~W} / \mathrm{cm}^{2}$ and inner constant cooling resulting the uniform inner surface temperature field $T_{i n n, \text { steel }}^{R \text { test }}=217.45^{\circ} \mathrm{C}$. Fig. $7 \mathrm{~b}$ shows the difference temperature field $\left(T_{\text {out,foil }}^{R \text { test }}-T_{\text {inn,steel }}^{R \text { test }}\right)$, which represents the product of constant thermal resistance and applied heat flux. This constant temperature field has been used directly in denominator in Eq.6.1.for correction of all records of phases \#2,\#3 and \#4 of presented case in form of already emissivity corrected, temperature difference field $\left(T_{\text {out }, \text { foil }}-T_{\text {bulk }}\right)$. 
Fig.8 shows a sequence of process for phase \#2 according Eq.6.1. Fig.8a shows the emissivity corrected temperature $T_{\text {out }, \text { foil }}$ field distribution. Fig.8b shows the temperature difference $\left(T_{\text {out,foil }}-T_{\text {bulk }}\right)$ field distribution. Consequently the calculation of the difference of temperature differences fields $\left(T_{\text {out foil }}-T_{\text {bulk }}\right)-\left(T_{\text {out }, \text { foil }}^{\text {Rtest }}-T_{\text {inn } \text {, steel }}^{\text {Rtel }}\right)$ i.e. the whole denominator of Eq.6.1, is shown in Fig.8c. Finally the absolute HTC values are pictured in Fig.8d as the HTC distribution field.

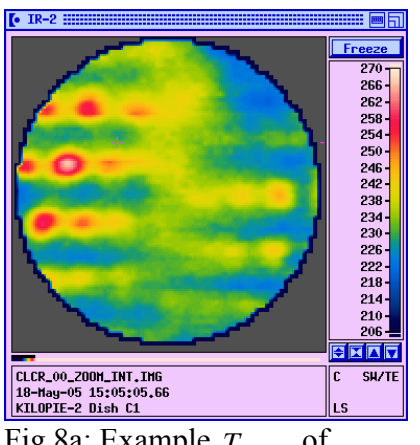

Fig.8a: Example $T_{\text {out }, \text { foil }}$ of

outer temperature field (emissi vity corrected) during phase \#2

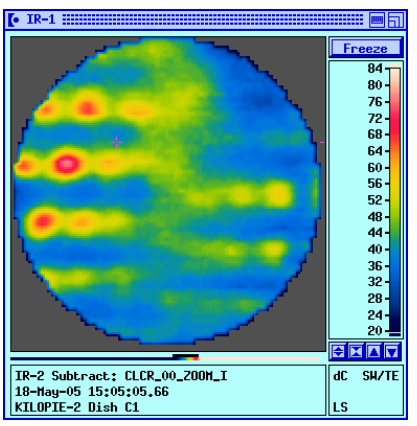

Fig.8b: Temperature differrence field $\left(T_{\text {out foil }}-T_{\text {bulk }}\right)$ from Eq.6.1 during phase \#2

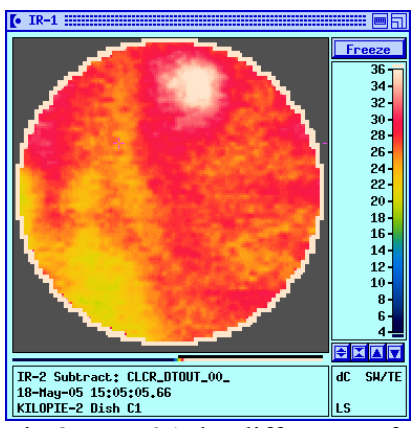

Fig.8c: Eq 6.1, the difference of temperature difference fields,

$\left(T_{\text {out }, \text { foi }}-T_{\text {bulk }}\right)-\left(T_{\text {out }, \text { foi }}^{\text {R test }}-T_{\text {inn }, \text { steel }}^{R \text { test }}\right)$

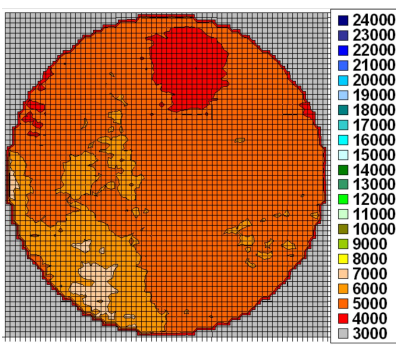

Fig.8d: Heat transfer coefficient distribution field within the phase \#2; HTC in W/ $\mathrm{Km}^{2}$

The sequence of steps in Fig.8a-d shows the strong capability of the 2DD IRT method to discover through visualization the swirl dead zone of flow velocity and consequently the bad cooling phenomena in phase \#2 of the presented case. Additionally it has to be mentioned that the dynamic capability of the IRT measurement allows study the behavior e.g. wandering of such dead zone or hot spot, with the time. The HTC values within the phase \#2 vary in the range 4000-7000 W/Km ${ }^{2}$ and are insufficient for the MEGAPIE target cooling. The switching on of the by-pass jet flow

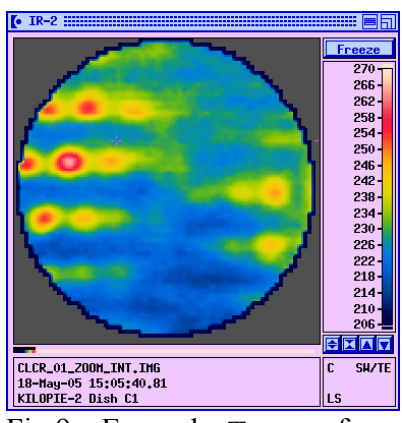

Fig.9a: Example $T_{\text {out,foil }}$ of

outer temperature field (emiss. cor.) during ph. \#2/\#3 change

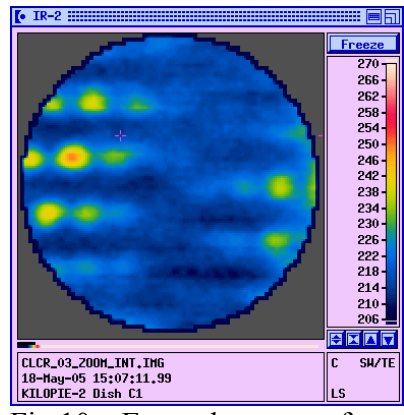

Fig.10a: Example $T_{\text {out }, \text { foil }}$ of

outer temperature field (emissi vity corrected) during phase \#3

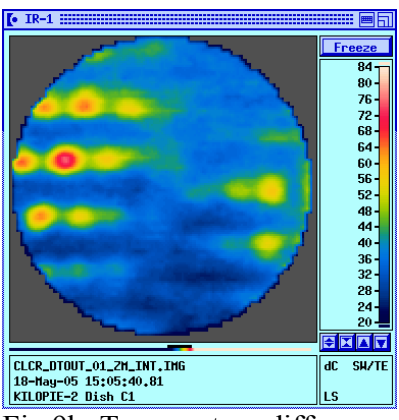

Fig.9b: Temperature differrence field $\left(T_{\text {out, foil }}-T_{\text {bulk }}\right)$ from Eq.6.1 during phase \#2/3

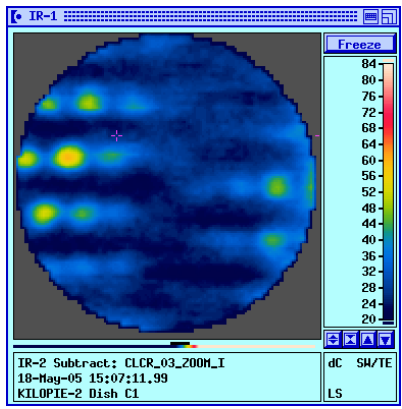

Fig.10b:Temperature differrence field $\left(T_{\text {out }, \text { foil }}-T_{\text {bulk }}\right)$ from Eq.6.1 during phase \#3

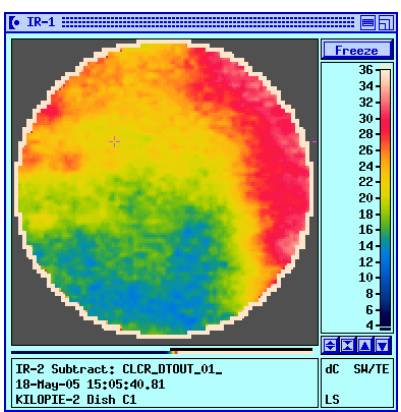

Fig.9c: Eq.6.1,the difference of temperature difference fields $\left(T_{\text {out }, \text { fol }}-T_{\text {bulk }}\right)-\left(T_{\text {out }, \text { fol }}^{\text {R test }}-T_{\text {inn }, \text { steel }}^{\text {Rtest }}\right)$

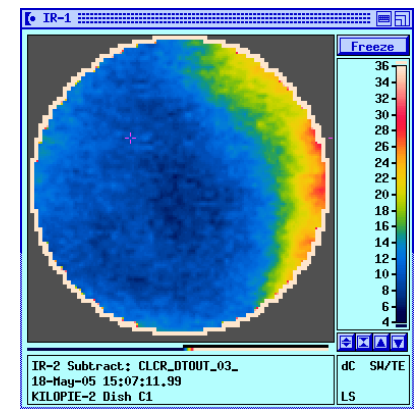

Fig.10c:Eq.6.1, difference of temperature difference fields $\left(T_{\text {out }, \text { fol }}-T_{\text {bulk }}\right)-\left(T_{\text {out }, \text { fol }}^{\text {R tes }}-T_{\text {inn, steel }}^{\text {R test }}\right)$

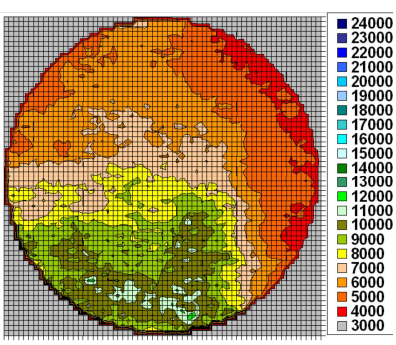

Fig.9d: HTC distribution field at the moment of phase \#2/\#3 change; HTC in $\mathrm{W} / \mathrm{Km}^{2}$

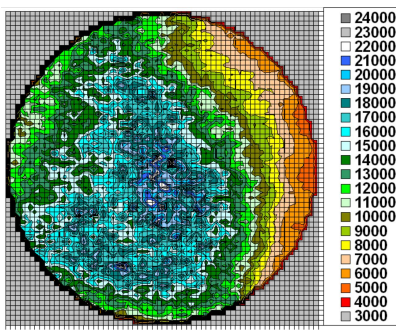

Fig.10d: Heat transfer coefficient distribution field within the phase \#3; HTC in $\mathrm{W} / \mathrm{Km}^{2}$ 
drastically changed this cooling situation. The similar sequence of process as in Fig.8 are constructed for two others characteristic moments of the case. The moment of the change from phase \#2 to \#3 is shown in Fig.9 and the fully deve loped by-pass jet flow of the phase \#3 in Fig.10. In phase \#3 we observe a rise of the HTC in wide central region over $16000 \mathrm{~W} / \mathrm{Km}^{2}$, which is sufficient for cooling of the MEGAPIE target. The top values of HTC reach $24000 \mathrm{~W} / \mathrm{Km}^{2}$.

The most important step, which allow immediately recognized the changes in the cooling situation within the processing sequence for each phase and between the phases, is transfer from outer surface (Figures $8 \mathrm{~b}, 9 \mathrm{~b}, 10 \mathrm{~b}$ ) to the inner surface (Figures 8c, 9c, 10c) temperature treatment of the cooling phenomena. Additionally the $20 \mathrm{~Hz}$ dynamic behavior of the flow effects in boundary layer are shown in an animation during the presentation of the paper.

Fig. 11 shows summarizing diagram based on 28 load cases

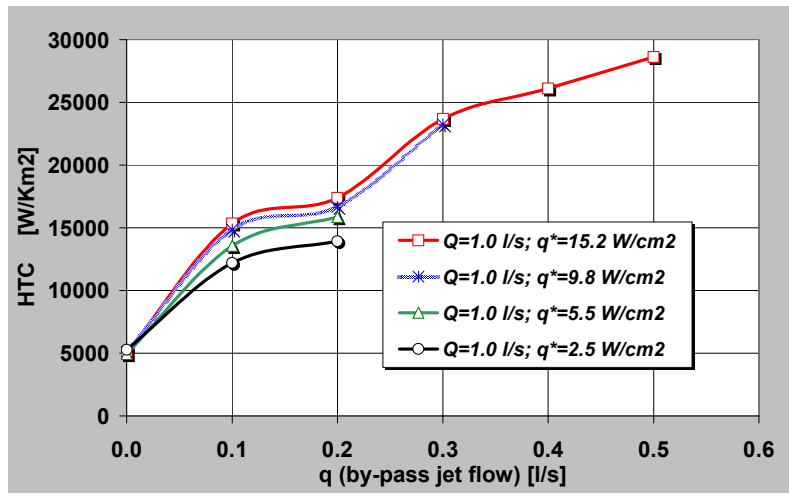

Figure 11: $H T C$ vs. by-pass jet flow $q$ and constant main flow $Q=1.0 \mathrm{l} / \mathrm{s}$ for different heat fluxes (power densities) $q^{*}$ performed within IRT KILOPIE experiment. The HTC at the central point of the sensor dish, for a set of flows parameters and heat fluxes, have been determinate in the same way as describe above. In general the very good cooling with bypass jet flow has been found and the predominant role of the rising linear velocity of the jet flow on the inner surface of the target window has been measured. An influence of rising heat flux on HTC values has been found. Higher heat flux yields higher HTC values, what represent conservative conditions for the real MEGAPIE heat flux approximated up to $125 \mathrm{~W} / \mathrm{cm}^{2}$. Is interesting to mention, that the real minimum of the cooling from Fig. $8 \mathrm{~d}\left(\mathrm{HTC}=3989 \mathrm{~W} / \mathrm{Km}^{2}\right)$ cannot be found for the measurement at central point only, and the advantage of the IR thermography measurements as an imaging techniques is quite evident.

\section{CONCLUSION}

The realization of a multilayer specimen sensor, which can create and apply a known heat flux, has allowed using the 2DD IRT method, the dynamic visualization of the cooling of the target proton beam window and the determination of the absolute values field of the heat transfer coefficient at steel-liquid metal interface.

\section{REFERENCES}

1. J.A. Patorski, G.S. Bauer and S. Dementjev, "Two-dimensional and dynamic (2DD) method of visualization of the flow characteristics in a convection boundary layer using infrared thermography", Proceedings of Thermosense XXII SPIE (ISSN 0277-786X) Vol. 4020, pp 240-251, 2000

2. J.A. Patorski, et al., "Two-dimensional and dynamic method of visualization of the flow characteristics in a convection boundary layer using infrared thermography", Journal of Theoretical and Applied Mechanics (ISSN 1429-2955), Vol. 39, pp 351-376, 2001

3. G.S.Bauer, M. Salvatores and G.Heusener, "MEGAPIE, a 1 MW Pilot Experiment for a Liquid Metal Spallation Target", J. Nucl. Mat. 296, pp.17-35, 2001

4. T. Dury, "Best-estimate fit for MEGAPIE Heat Deposition Profile Data of 19.2.01", PSI Memorandum, 22.03.2001 , PSI, CH-5232 Villigen, Switzerland, 22.03.2001

5. J.A. Patorski, et al., "Experimental estimation of optimum Bypass-jet-flow conditions for the cooling of the window of the SINQ liquid metal target", PSI Annual 2000, ISSN 1423-7350, Vol. VI, , PSI, CH-5232 Villigen, Switzerland pp.42-44, March 2001

6. AGEMA Infrared Sytems, "Thermovision ${ }^{\circledR 900}$ Series User's Manual" AGEMA Infrared Sytems Publication No.556960, 1993

7. F.P. Incorpera, D.P. DeWitt "Fundamentals of Heat and Mass Transfer" (Fourth Edition), John Wiley \& Sons, New York, 1996

8. J.A. Patorski, B.Sigg, I. Platnieks, F. Groeschel and R. Stieglitz "Results of IRT- and TC-KILOPIE-1 Experiments“, PSI Annual 2004, ISSN 1423-7326, Vol.III, p.204-205, March 2005

9. J.A. Patorski, F. Groeschel, F. Barbagallo and I. Platnieks, "First results of the KILOPIE-2 Pre-experiment", Proc. 6th Technical Review Meeting, MEGAPIE, SCK-CEN, Mol, Begium, 27-29 ${ }^{\text {th }}$ June 2005

10. J.A. Patorski, "MEGAPIE Integrated Test; Qualitative characteristic of th by-pass jet cooling Warm Jet-3

Experiment ", MIT Data Evaluation Meeting, PSI CH-5232 Villigen, Switzerland, $18^{\text {th }}$ January 2006 